Research Paper

\title{
Generation of VEGF knock-in Cashmere goat via the CRISPR/Cas9 system
}

\author{
Xiao Hu*, Fei Hao*, Xiaocong Li, Zhiyuan Xun, Yuan Gao, Bingxu Ren, Ming Cang, Hao Liang, Dongjun Liu ${ }^{凶}$ \\ State Key Laboratory of Reproductive Regulation \& Breeding of Grassland Livestock, Inner Mongolia University, Hohhot, 010000, China. \\ *These authors contributed equally to this work. \\ $\bowtie$ Corresponding author: Dongjun Liu, E-mail: nmliudongjun@sina.com; Tel: +86 471 3679865; Fax: +86 4714995071.
}

(C) The author(s). This is an open access article distributed under the terms of the Creative Commons Attribution License (https://creativecommons.org/licenses/by/4.0/). See http:/ /ivyspring.com/terms for full terms and conditions.

Received: 2020.11.06; Accepted: 2021.02.09; Published: 2021.03.02

\begin{abstract}
Cashmere is a rare and specialised animal fibre, which grows on the outer skin of goats. Owing its low yield and soft, light, and warm properties, it has a high economic value. Here, we attempted to improve existing cashmere goat breeds by simultaneously increasing their fibre length and cashmere yield. We attempted this by knocking in the vascular endothelial growth factor (VEGF) at the fibroblast growth factor 5(FGF5) site using a gene editing technology and then studying its hair growth-promoting mechanisms. We show that a combination of RS-1 and NU7441 significantly improve the efficiency of CRISPR/Cas9-mediated, homologous-directed repair without affecting the embryo cleavage rate or the percentages of embryos at different stages. In addition, we obtained a cashmere goat, which integrated the VEGF gene at the FGF5 site, and the cashmere yield and fibre length of this gene-edited goat were improved. Through next-generation sequencing, we found that the up-regulation of VEGF and the down-regulation of FGF5 affected the cell cycle, proliferation, and vascular tone through the PI3K-AKT signalling pathway and at extracellular matrix-receptor interactions. Owing to this, the gene-edited cashmere goat showed impressive cashmere performance. Overall, in this study, we generated a gene-edited cashmere goat by integrating VEGF at the FGF5 site and provided an animal model for follow-up research on hair growth mechanisms.
\end{abstract}

Key words: CRISPR/Cas9; VEGF; knock-in; cashmere goats; hair growth

\section{Introduction}

The emergence and development of gene editing have realised the site-directed transformation of genomic DNA sequences and promoted a revolution in the field of biomedicine. A pre-condition for gene editing is the double-strand break (DSB). However, the efficiency of traditional gene manipulation technology to achieve gene editing using donor plasmids and naturally generated DSB is very low, i.e. approximately $10^{-4}-10^{-6}$, which limits the application of this technology in gene function research, disease model construction, and genetic breeding [1-4]. Therefore, we need to artificially introduce a DSB.

Over the past few decades, from the restriction enzymes of the 1970s to today's advanced gene-editing techniques, the precise manipulation of eukaryotic genomes with molecular tools derived from the prokaryotic immune system has improved. Most notable is the introduction of clustered regulatory interspaced short palindromic repeat (CRISPR) editing techniques [5-7]. The CRISPR/ CRISPR-associated protein-9 nuclease (Cas9) system is a third-generation gene-editing technology known for its unprecedented specificity, effectiveness, and versatility [8]. It is derived from the acquired immune system of prokaryotes, providing acquired immunity against the invasion of foreign gene elements such as phages, and is widely found in archaea and other prokaryotes [9].

Compared with the traditional zinc-finger and transcription activator-like effector nucleases, the CRISPR/Cas9 system has higher gene-editing efficiency and lower production costs. It can be flexibly applied to different nucleotide sequences and is a more powerful gene-editing tool [10]. With the emergence, upgradation, and improvement of CRISPR/Cas9, the efficiency of DSB generation in the 
genome has become fully guaranteed, which provides favourable conditions for the development of gene-editing animal models.

The Inner Mongolian Albas cashmere goat is an excellent local cashmere and meat hybrid breed formed through long-term natural breeding. The cashmere produced is of high quality and has a high economic value, but the cashmere yield is low. Therefore, it is very important to cultivate new cashmere goat lines with higher yields. However, the conventional breeding technology has several disadvantages, such as the need for a large initial investment, slow effects, and long cycle. The breeding of new varieties with high yields, high qualities, and other excellent traits is thus progressing relatively slowly. Therefore, gene-editing technology should be used to cultivate new cashmere goat strains with high yields.

Vascular endothelial growth factor (VEGF) is an active and widely distributed growth factor secreted by vascular endothelial cells. It can increase vascular permeability and plays an irreplaceable role in angiogenesis and mesenchymal cell differentiation [11-13]. Studies have shown that, after binding to its receptor VEGFR-2, VEGF can promote hair growth by stimulating cell proliferation and regulating the hair growth cycle [14, 15].

Hair growth in mammals shows seasonal and periodic changes in anagen, catagen, and telogen content [16]. Therefore, prolonging the anagen phase can improve the quality and yield of hair. The hair growth cycle is regulated by a series of cell growth factors [17]. As fibroblast growth factor (FGF)-5 is a signalling protein secreted during the hair growth cycle, mutations in mammalian FGF5 can prolong the anagen phase and lead to new long-hair traits [18-23].

It has been reported that embryos microinjected with CRISPR/Cas9 can be used to obtain mosaic goats with FGF5 gene disruption, which increase the length of the cashmere fibres in these cashmere goats [24]. However, the stable inheritance of qualified characteristics is essential in the genetic breeding of domestic animals. Therefore, in this study, we used somatic cell nuclear transfer (SCNT) to prepare a gene-edited cashmere goat which can transmit superior traits to the next generation.

In conclusion, we here attempted to combine CRISPR/Cas9 and SCNT to generate gene-edited cashmere goats that integrate VEGF at the FGF5 site and promote VEGF overexpression through FGF5 knockout. The results of this study provide a basis for the efficient generation of gene knock-in animals as well as an animal model for studying the hair growth-promoting mechanisms of cashmere goats.

\section{Materials and Methods}

\section{Ethics statement}

All experiments performed followed the National Research Council Guide for the Care and Use of Laboratory Animals. All protocols were approved by the Institutional Animal Care and Use Committee of Inner Mongolia University. All animals were maintained at the Inner Mongolia YiWei White Cashmere Goat Limited Liability Company.

\section{Cell culture}

Goat foetal fibroblast cells (GFFs) were isolated from goat embryos at 40 days. The cells were maintained in Dulbecco's Modified Eagle's Medium (DMEM): nutrient mixture F-12 (Gibco, Carlsbad, USA), supplemented with $15 \%$ foetal bovine serum (FBS, Biological Industries, Kibbutz Beit Haemek, Israel). The culture was kept in an incubator with 5\% $\mathrm{CO}_{2}$ at $37^{\circ} \mathrm{C}$. Cells in the log phase were seeded into six-well plates. The RAD51-stimulatory compound, RS-1 [25] (10 $\mu \mathrm{M}$, Selleck, Houston, USA), and the DNA-PKcs inhibitor, NU7441 [26] (2 $\mu \mathrm{M}$, Selleck), dissolved in dimethyl sulfoxide, were diluted with the cell culture medium.

\section{Cell cycle analysis}

The cell cycle was measured using the Cell Cycle and Apoptosis Analysis Kit (7sea Biotech, Shanghai, China). Cell suspensions were prepared from GFFs treated with inhibitors in pre-cooled phosphatebuffered saline (PBS, Gibco). After an overnight fixation with $70 \%$ ethanol at $4{ }^{\circ} \mathrm{C}$, the cells were stained with $500 \mu \mathrm{L}$ of $2.5 \%$ propidium iodide (PI) solution at $37{ }^{\circ} \mathrm{C}$ for $30 \mathrm{~min}$ away from light. Flow cytometry (BD Biosciences, New York, USA) was performed within $5 \mathrm{~h}$, and the excitation wavelength was $488 \mathrm{~nm}$.

\section{Apoptosis analysis}

Apoptotic cells were detected using the Annexin V-FITC/PI Apoptosis Analysis Kit (7sea Biotech). GFFs were inoculated in 24-well plates, in triplicate, and cultured for $24 \mathrm{~h}$. After the addition of inhibitors, the culture was continued for $24 \mathrm{~h}$. The culture solutions were then collected in centrifuge tubes because they contained apoptotic cells. Then, cells in each group were trypsinised and washed with PBS to completely remove the trypsin. Subsequently, the cells were re-suspended in $400 \mu \mathrm{L}$ of binding buffer. V-fluorescein isothiocyanate $(5 \mu \mathrm{L})$ was added to the wells and mixed by gentle swirling. The solutions were then incubated at $20^{\circ} \mathrm{C}$ for $15 \mathrm{~min}$ away from light. The cells were stained with $10 \mu \mathrm{L}$ of PI and incubated at $4{ }^{\circ} \mathrm{C}$ for $5 \mathrm{~min}$ in the dark. Finally, the 
apoptosis rate of the cells was detected by flow cytometry for $30 \mathrm{~min}$. The FITC and PI were detected with $488 \mathrm{~nm}$ and $575 \mathrm{~nm}$ excitation light, respectively.

\section{DNA replication activity detection}

5 Ethynyl 2 'deoxyuridine (EdU) is a thymidine nucleoside analogue that can replace thymine in replication during cell proliferation [27]. Using the specific reactions between EdU and Apollo fluorescent dyes, the DNA replication activity of cells can be rapidly and accurately detected. EdU assays are faster, more sensitive, and more accurate than BrdU assays [28]. Cells in logarithmic growth stage were inoculated in 96-well plates with $10^{5}$ cells per well and cultured to the normal growth stage for medical treatment. Each well received $100 \mu \mathrm{L}$ of 50 $\mu \mathrm{M}$ EdU (Cell-Light ${ }^{\mathrm{TM}}$ EdU Apollo643 In vitro Kit, RiboBio, Suzhou, China) at 1:1000 dilution in 15\% cell medium, and the samples were then incubated at room temperature for $2 \mathrm{~h}$. The culture medium was discarded, and the plate was washed twice with PBS. The cells were fixed at room temperature for $30 \mathrm{~min}$ with $4 \%$ paraformaldehyde. The stationary liquid was discarded, $50 \mu \mathrm{L}$ of $2 \mathrm{mg} / \mathrm{mL}$ glycine was added to each well, and the samples were incubated in a decolourising shaker for $5 \mathrm{~min}$. The glycine solution was then discarded, and the plate was washed with PBS for 5 min. Next, $100 \mu \mathrm{L}$ of $0.5 \%$ Triton X-100 was added to each well and incubated for $10 \mathrm{~min}$. Apollo staining solution $(100 \mu \mathrm{L})$ was added to each well, and the samples were incubated at room temperature for 30 min away from light. The Apollo staining solution was discarded, and the plate was washed with $0.5 \%$ Triton X-100 for $30 \mathrm{~min}$. Then, $100 \mu \mathrm{L}$ Hoechst 33342 reaction mixture was added to each well and the solutions incubated at room temperature for $30 \mathrm{~min}$ away from light. The Hoechst 33342 reaction mixture was discarded, and the plate was washed with PBS three times. Ultimately, the cell staining results were observed under a laser confocal microscope (NIKON, Tokyo, Japan).

\section{Detection of knock-in efficiency}

We constructed a protomerless DsRed2 reporter vector to determine the effect of small molecule inhibitors on homologous-directed repair (HDR) efficiency. For the FGF5 locus, the DsRed2 reporter vector consisted of a 926-bp 3'-homology arm (HA), a DsRed2 coding sequence, and a 1099-bp 5'-HA (Figure 2A). Twelve hours after electroporation transfection of GFFs, the small molecule inhibitors were added to the cell culture medium and treated for $24 \mathrm{~h}$. After changing the normal culture medium, the culture continued for 24 hours and fluorescenceactivated cell sorting (FACS) was used to analyse the proportion of red fluorescent cells (Figure 2C).

\section{Cell electroporation transfection}

To avoid the toxicity of Lipofectamine to cells, the DsRed2 reporter vector, or the VEGF-targeted integration plasmid and Cas9/gRNA co-expression plasmid, were transfected into the GFFs by electroporation transfection (Super Electroporator NEPA21 In vitro \& In vivo Electroporation, Nepa Gene, Chiba, Japan). The sequences and locations of target sites were shown in Figure 2F. The small molecule inhibitor-treated cells were adjusted to a concentration of $10^{6}$ cells per $100 \mu \mathrm{L}$. The electroporation transfection parameters were as follows: voltage $225 \mathrm{~V}$, pulse length $2.5 \mathrm{msec}$, pulse interval $50 \mathrm{msec}$, number of pulses 4 , decay rate $10 \%$, and positive polarity. The cell suspension was transferred to an incubator with $5 \% \mathrm{CO}_{2}$ at $37^{\circ} \mathrm{C}$.

\section{Production of knock-in cell colony}

In consideration of the biosafety issues involved in gene-editing animals and the subsequent industrialisation of varieties, neither resistance screening nor fluorescent proteins were used in the screening of the knock-in cell colonies. To improve the efficiency of the integration of the VEGF gene and avoid the kanamycin resistance gene integrating into the cashmere goat genome, we used the restriction enzymes SfiI and PciI to linearise the VEGF-targeted integration plasmid, and recovered large fragments containing the upstream HAs, skin tissue specific-promoter keratin-associated protein (Kap) 6.1, the VEGF gene, PolyA, and the downstream HAs using agarose gel electrophoresis. After transfecting the main frame of the VEGF-targeted integration plasmid and the Cas9/gRNA co-expression plasmid into the GFFs, single cells were inoculated with flow cytometry into 96-well plates containing DMEM/F12 medium supplemented with $15 \%$ FBS. After approximately 15 days, the growth confluence of the single-cell clones reached $80-90 \%$, and were transferred to 24-well plates for further culture. When the cells had grown to $80 \%$ in the 24 -well plates, they were trypsinised. A part of the cell suspension was further cultured in the well, and the remaining was extracted for genotyping. The genomes of the cell colonies were extracted using the Wizard ${ }^{\circledR}$ Genome DNA Purification Kit (Promega, Madison, USA), following the supplier's protocol. The polymerase chain reactions (PCR) identification primer locations are shown in Figure 2A, and the sequences are shown in S1. Knock in cell colonies of the GFFs were selected as donor cells for subsequent SCNT. 


\section{Off-target analysis}

To ensure the safety of the gene-edited goats, we designed sgRNA for FGF5 exon 1, and predicted all the potential off-target sites (five mismatches within the 20-nucleotide sgRNA) of this sgRNA in the goat genome, using the CCTop-CRISPR/Cas9 target online predictor [29]. We then conducted PCR and sequencing on the 10 sites with high off-target probability to detect whether off-target attachment occurred in those locations. The primer sequences are shown in S1.

\section{Somatic cell nuclear transfer and embryo transplantation}

The ovaries of cashmere goats were mechanically dissected and the cumulus-oocyte complexes were collected and cultured in M-199 maturation medium (Hyclone) in an incubator with $5 \% \mathrm{CO}_{2}$ at $38.5^{\circ} \mathrm{C}$ for $18 \mathrm{~h}$. The cumulus-oocyte complexes were digested in $0.1 \%$ hyaluronidase solution, and the cumulus cells on the surfaces of mature oocytes were completely removed. After the nuclei of the mature oocytes were removed, the donor cells were microinjected into the perivitelline spaces of the enucleated oocytes in cytochalosin B droplets. Subsequently, fusion was performed with ECM 2001 Electro Cell Manipulator (Harvard Apparatus, Cambridge, USA). The reconstructed embryos were pre-treated in synthetic oviductal fluid with amino acids (SOFaa solution) containing 5 M IA23187 for 5 min and then transferred to SOFaa solution containing $2 \mathrm{mM}$ 6-dimethylaminopurine. The embryos were cultured in an incubator at $38.5^{\circ} \mathrm{C}$ with $5 \% \mathrm{CO}_{2}$ for $3.5 \mathrm{~h}$ for activation. The reconstructed embryos were then cultured in an in vitro development solution at $38.5^{\circ} \mathrm{C}$ for $48 \mathrm{~h}$. We selected embryos that had developed to the two-cell, four-cell, and eight-cell stages and surgically transferred them into the oviducts of recipient cashmere goats in oestrus, with each recipient receiving 3-4 embryos.

\section{Southern blot hybridization}

For Southern blotting, $3 \mu \mathrm{g}$ of DNA, extracted from cashmere goats and digested by XhoI and NdeI, was electrophoresed in $1.5 \%$ agarose gel and transferred to nylon membranes by capillary transfer. PCR amplification was performed using a VEGF-targeted integration plasmid as a template, and the probe fragment was purified after agarose gel electrophoresis. The location of the probe is shown in Figure 2A, and its primer sequences are shown in S1. The probe was combined with digoxigenin for hybridisation. Hybridisation was conducted at $42{ }^{\circ} \mathrm{C}$ for $16 \mathrm{~h}$. The hybridised probes were immunodetected using anti-digoxigenin-alkaline phosphatase and visualised with the chemiluminescence substrate CSPD (Roche, Basel, Switzerland).

\section{Skin tissue paraffin sectioning \& HE stain}

The goat skin tissues were fixed in $4 \%$ paraformaldehyde for $24 \mathrm{~h}$, washed under running water, dehydrated using graded ethanol, vitrificated with dimethylbenzene, embedded in paraffin, and sectioned $(7 \mu \mathrm{m})$. After dewaxing the sections with xylene, they were rehydrated with ethanol and rinsed with distilled water to remove the residual xylene and ethanol. The cell nucleus was stained with haematoxylin for $10 \mathrm{~min}$, and then the cytoplasm was stained with eosin for $30 \mathrm{~s}$. After gradient dehydration with ethanol and dimethylbenzene, neutral balsam was added to the sections before covering them with a microscope coverslip.

\section{Western blotting}

The Mammalian Protein Extraction Kit (CWBIO, Beijing, China) was used to extract cashmere skin proteins, and the concentration of the protein samples was measured using the PierceTM BCA Protein Assay Kit (Thermo Scientific) and a Varioskan Flash reader (Thermo Scientific, Thermo Scientific, Waltham, USA). Subsequently, sodium dodecyl sulphate-polyacrylamide gel electrophoresis and transmembrane analysis were carried out using PowerPac Basic (Bio-Rad, Berkeley, USA) and PowerPac HC ${ }^{\mathrm{TM}}$ (Bio-Rad). Tris Buffered (CWBIO, Beijing, China) saline Tween was used to wash the nitrocellulose filter membrane for $5 \mathrm{~min}$. After blocking the membranes with $5 \%$ skim milk (Difco ${ }^{\mathrm{TM}}$ Skim Milk, BD Biosciences) for $2 \mathrm{~h}$, alpha-tubulin antibody (Proteintech, Wuhan, China), FGF5 antibody (Proteintech, Wuhan, China), and VEGF antibody (Proteintech) were added at a dilution of 1:1000 in 5\% bovine serum albumin, and the samples incubated at $4{ }^{\circ} \mathrm{C}$ overnight. After washing-off the excess primary antibodies with TBST, the secondary antibody (Horseradish peroxidase-conjugated affinipure goat anti-rabbit IgG, Proteintech) was added at a dilution of $1: 1000$ in $0.5 \%$ bovine serum albumin and the samples incubated for $1 \mathrm{~h}$ at room temperature. The membrane was washed three times with TBST to remove the secondary antibody. Finally, Pierce ${ }^{\mathrm{TM}}$ ECL Western Blotting Substrate (Thermo Scientific) was used for colour rendering, and the Tanon-5200 image-analysis system (Tanon, Shanghai, China) was used to visualise the protein bands.

\section{Real-time polymerase chain reaction}

Total RNA was extracted from the skin tissue of cashmere goats using RNAiso Plus (TaKaRa Bio, Shiga, Japan). The integrity and molecular weight of the RNA were checked using denaturing agarose gel 
electrophoresis. The RNA concentration was determined using a Nanodrop 2000 spectrophotometer (Thermo Fisher). The cDNA was prepared from $500 \mathrm{ng}$ of RNA using the PrimeScript ${ }^{\mathrm{TM} R T}$ Reagent Kit (TaKaRa Bio), following the supplier's protocol. Quantitative real-time PCR was performed with SYBR Premix Ex Tap ${ }^{\mathrm{TM} I I}$ (TaKaRa Bio), and the relative expression of the target gene was calculated following the $2-\Delta \Delta \mathrm{Ct}$ method. The names and sequences of the primers are provided in S2.

\section{Quality and yield of cashmere}

Cashmere quality and yield are important factors for measuring economic benefits. All the cashmere of the three goats was collected and weighed. The fibre was smashed and its fineness measured using a BT Benchtop mass spectrometer (OFDA, Sydney, Australia). Fracture strength, breaking strength, and elongation at break were measured using the Yg006 Electronic Single fibre Strength Tester (Yanshuo, Xian, China). We used a fibre diagram machine (Wira Instrumentation, Bradford, England) to randomly select fibres to measure length.

\section{Multiple omics next generation sequencing}

Skin tissues of cashmere goats were collected as samples for transcriptome, proteome, and metabolome studies. Eukaryotic mRNA sequencing was based on the Illumina Novaseq 6000 (Illumina, San Diego, USA) sequencing platform. The clean reads of each sample were compared with the designated reference genome. Based on the quantitative results of the expression levels, the differentially expressed genes (DEGs) between two groups were analysed. The difference analysis software used was edgeR, and the screening threshold was $|\log 2 \mathrm{FC}| \geq 0.848$ \& padjust $<0.05$. In order to exclude the possible influence of somatic cell nuclear transplantation on individual goats, SCNT and WT were used as the control group and compared with GEC in the subsequent analysis.

The instrument used for proteome mass spectrometry was the Q_Exactive HF-X (Thermo Scientific). A UPLC-TripleTof system (AB SCIEX, WOODLANDS, Singapore) was used for liquid chromatography-mass spectrometry non-targeted metabolome sequencing. The Gene Ontology (GO) enrichment and Kyoto encyclopedia of genes and genomes (KEGG) enrichment were analysed and visualised on the free online Majorbio Cloud Platform.

\section{Statistical analysis}

The results are presented as mean \pm standard deviation (SD). Student's t-tests were carried out to analyse the differences in the measurement data. A P-value $<0.05$ was taken to indicate statistical significance.

\section{Results}

\section{RS- 1 and Nu-7441 treatment enhances CRISPR/Cas9 mediated knock-in efficiency in GFFs}

The cell colony of VEGF integrated at FGF5 site was used for subsequent SCNT, which was derived from the proliferation of a single cell and required high cell status and quantity. Therefore, we hope to reduce the adverse effects on cells while improving HDR. Therefore, $24 \mathrm{~h}$ after the GFFs were treated with different small molecules, the cell cycle, apoptosis, and DNA replication activity were respectively detected. Using NU7441 alone had a great effect on the cell cycle (Figure 1A). In addition, NU7441 increased the number of apoptotic cells (Figure 1B, E). Because the inhibitors added were all related to DNA repair, we also examined DNA replication activity. Similar to apoptosis, RS-1 had the least effect on DNA replication (Figure 1C).

To ensure that the trends in the experimental results were consistent, the sequence and length of the HA of the DsRed2 reporter vector were identical to those of the VEGF site-directed integration vector. By means of FACS, we found RS-1 and Nu-7441 treatment increased HDR efficiencies by 3-4 times (Figure 1D, F) and had less effect on cell cycle, apoptosis, and DNA replication activity.

\section{Production of knock-in cell colony}

Flow cytometry was used to inoculate the single cells into 96-well plates for culture. After approximately 15 days, the cells were subcultured and identified (Figure 2E). The genomic DNA of cell colonies was extracted for PCR identification, and the partial electrophoresis results were shown in Figure 2D. The sequencing results confirmed that seven cell colonies achieved VEGF knock-in. To ensure the efficiency of the targeted integration of the VEGF gene, we transferred the Cas9/gRNA co-expression plasmids into GFFs, instead of Cas9 mRNAs or proteins, which increased the risk of off-target attachment to some extent. PCR and sequencing of 10 potential off-target sites (Figure 2B) showed that none of them contained mutations.

\section{Effect of inhibitors treatment on embryo cleavage}

A total of 1,824 oocytes were collected from 463 ovaries through mechanical cutting, and 936 mature oocytes were obtained after $18 \mathrm{~h}$ of in vitro culture, 
with a maturity rate of $51.3 \%$. The mature oocytes were used for SCNT (Figure 3A). After 48 h, embryo cleavage had developed to different stages (Figure 3B). The donor cells used for SCNT have no significant effect in the embryo cleavage rates or

A

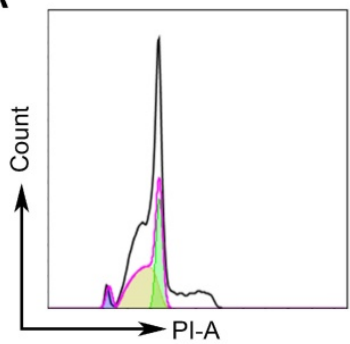

RS-1

B

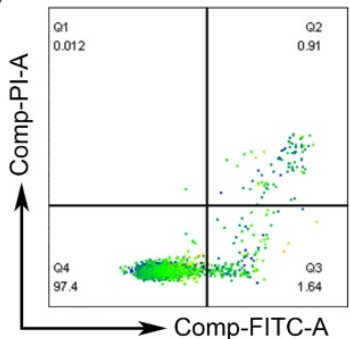

RS-1

C

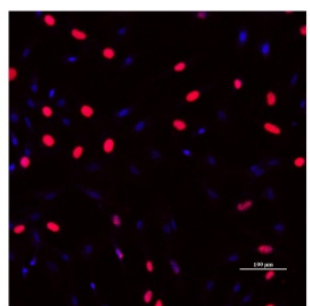

RS-1

D

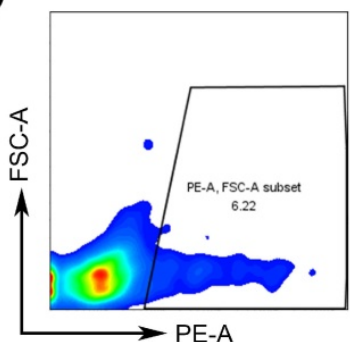

RS-1

$E$

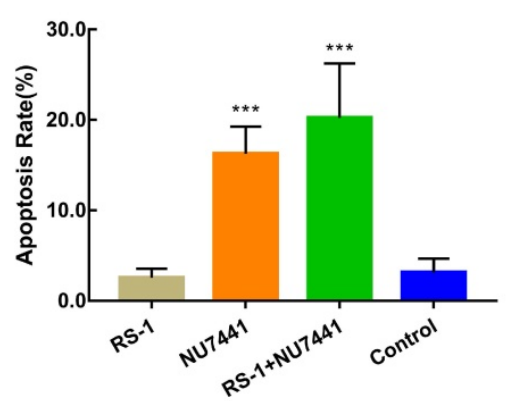

percentages of embryos at different stages after being treated with small molecules (Figure 3C, D). The results showed that RS-1 and NU7441 treatment had no adverse effects on embryos.

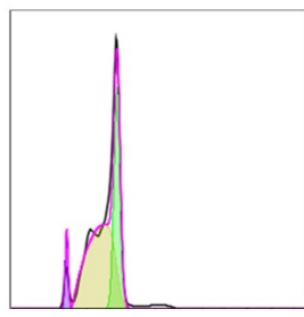

RS-1+NU7441

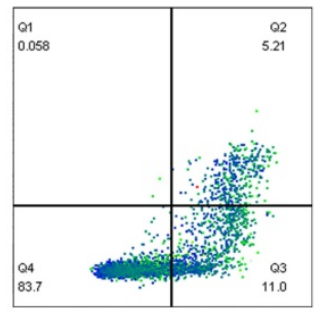

NU7441

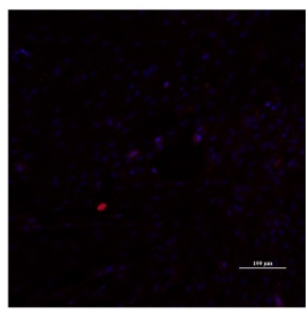

NU7441

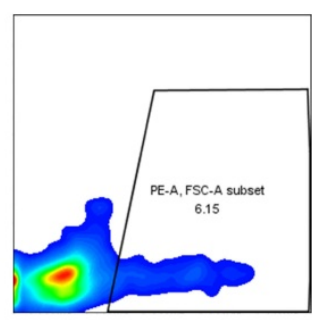

NU7441

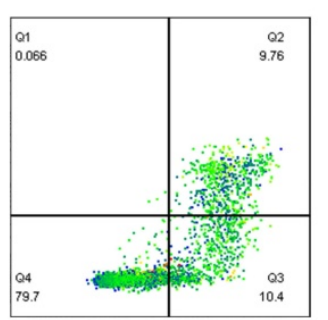

RS-1+NU7441

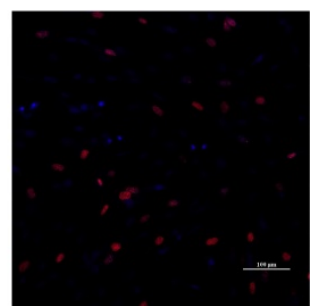

RS-1+NU7441

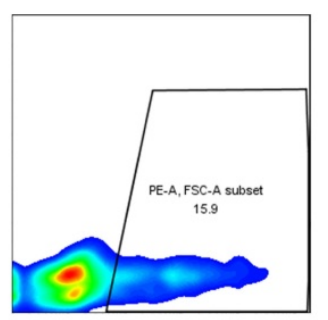

RS-1+NU7441

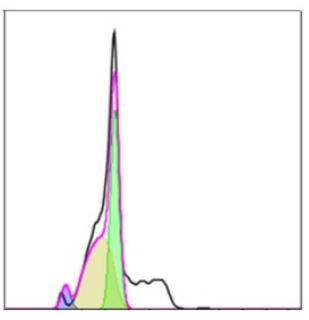

Control

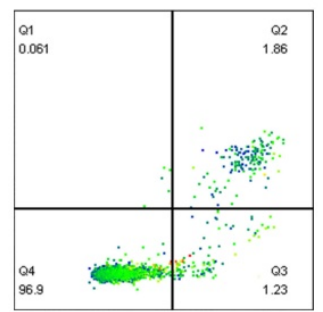

Control

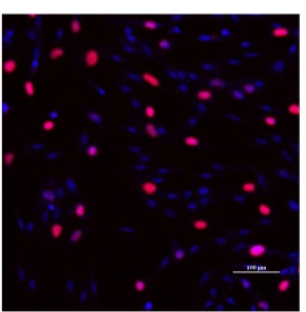

Control

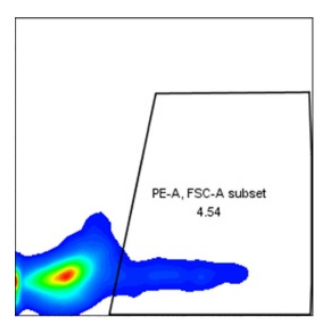

Control
F

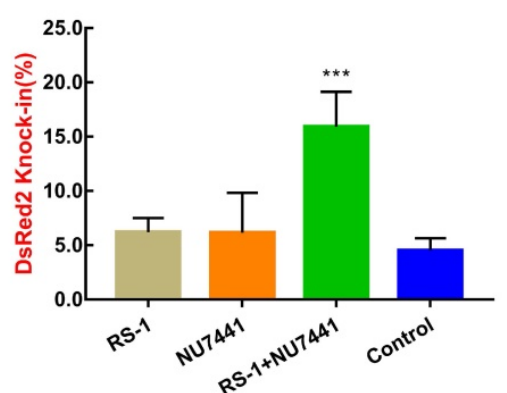

Figure 1. Effects of small molecules on cell cycle, apoptosis, proliferation, and HDR efficiency. A. Cell cycle after treatment with small molecules. B. Cell apoptosis. C. Cell proliferation and toxicity assay. D. HDR efficiency of DsRed2 knock-in. E. Cell apoptosis statistics. F. HDR efficiency statistics of DsRed2. 
A Cas9 target sequence : AGCCCCTCGGGGCGCCGGACCGG

DsRed2 reporter HA SA-DsRed2 HA

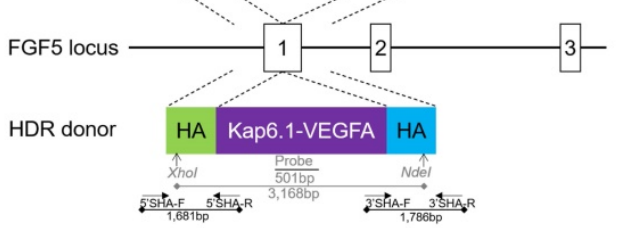

C

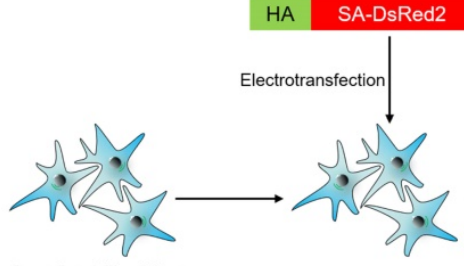

Goat fetal fibroblasts

E

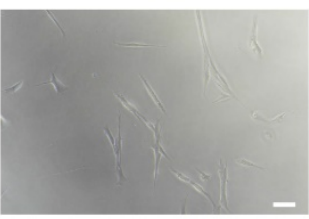

3 days

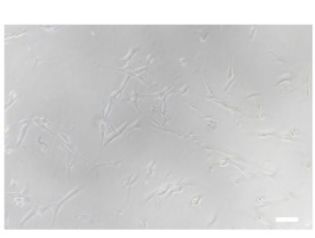

7 days

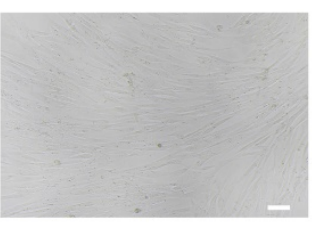

15 days

\begin{tabular}{|l|l|c|l|c|c|}
\hline \multicolumn{1}{|c|}{ Gene ID } & $\begin{array}{c}\text { Gene } \\
\text { Name }\end{array}$ & Type & Target_seq & PAM & MM \\
\hline ENSCHIG00000024886 & FGF5 & Exonic & AGCCCCTC [GGGGCGCCGGAC] & CGG & 0 \\
\hline ENSCHIG00000001632 & & Intronic & GGACCATC [GGGGCGCCGGAC] & CGG & 3 \\
\hline ENSCHIG00000021838 & EEFSEC & Intergenic & GGGCCGCC [GGGGGCCGGAC] & GGG & 4 \\
\hline ENSCHIG00000015061 & TNRC18 & Intronic & GGCCACAC [GGGCCGCCGGAC] & CGG & 4 \\
\hline ENSCHIG00000018282 & PIM3 & Exonic & CGCCGCGC [GGGGAGCCGAC] & CGG & 4 \\
\hline ENSCHIG00000024318 & PRKCA & Intergenic & AGGCCCCC [GGGGCTCGGAC] & CGG & 3 \\
\hline ENSCHIG00000021893 & HCN2 & Exonic & GCCCCCTC [CGGGCCCGGAC] & CGG & 4 \\
\hline ENSCHIG00000025441 & ASIC5 & Intronic & TGCCACTT [GGGGCTCCGGAC] & TGG & 4 \\
\hline ENSCHIG00000025391 & NOS1 & Exonic & AGCCGCGC [GGGGGTCGGAC] & CGG & 3 \\
\hline ENSCHIG00000013028 & ZNF48 & Intergenic & AGCTCCGG [GGGGCCCGGAC] & TGG & 4 \\
\hline ENSCHIG00000018512 & ALG12 & Exonic & TGCCACTG [GGGGCGGGGAC] & AGG & 4 \\
\hline
\end{tabular}

D

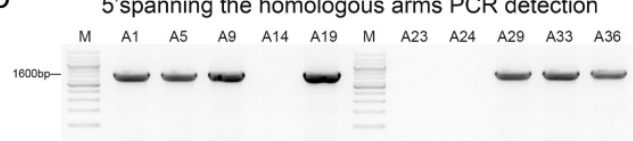

3'spanning the homologous arms PCR detection M A1 A5 A9 A14 A19 M A23 A24 A29 A33 A36

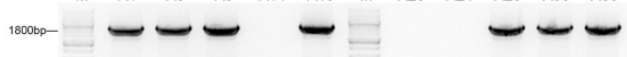

F

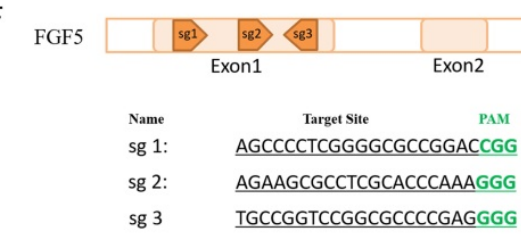

Figure 2. Production of Knock-in cell colony. A. Gene-targeting strategy of Cas9-mediated knock-in of VEGF or DsRed2 to the FGF5 locus. B. Gene names, types, and target sequences of the top 10 potential off-target sites of sgRNA were used in this study. C. Process of exploring the optimal HDR efficiency of small molecules. D. Spanning the homologous arms PCR identification results of cell colonies. E. Images of cell colonies at 3, 7, and 15 days. Scale bar $=100 \mu \mathrm{m}$. F. The sequences and locations of target sites at exon 1 of FGF5 gene.
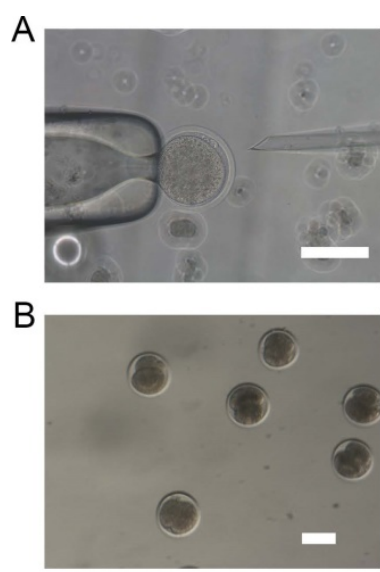

2-cell stage embryo

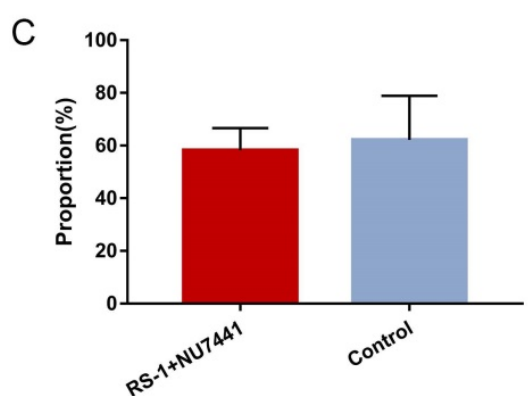

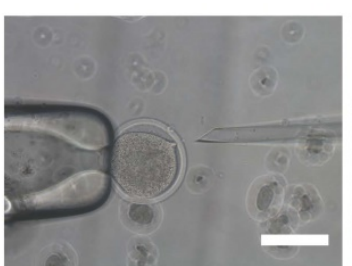
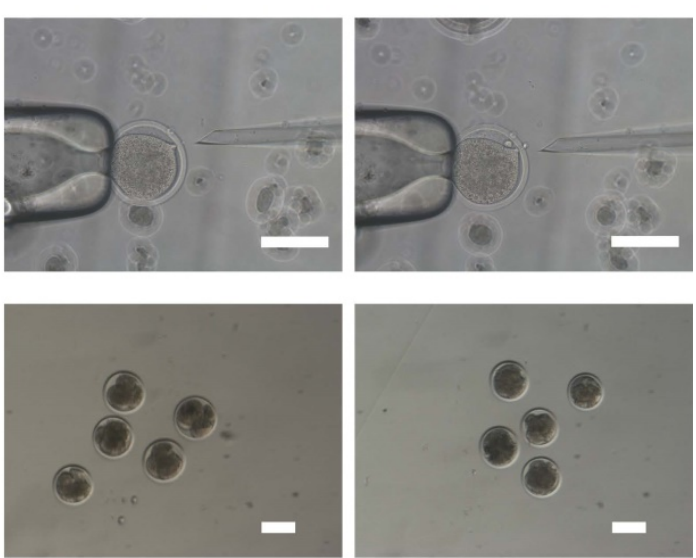

4-cell stage embryo

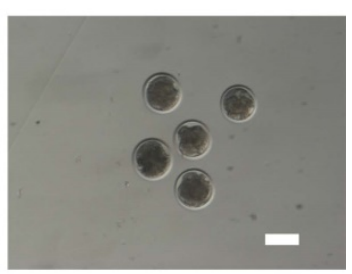

8-cell stage embryo

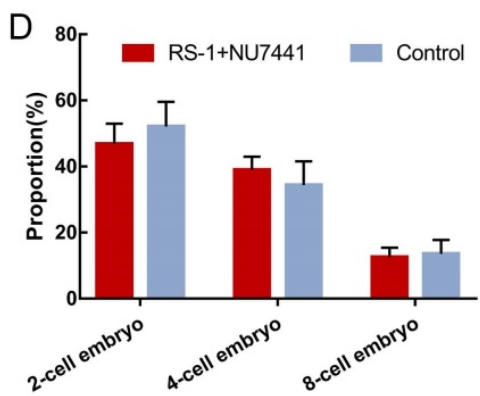

Figure 3. Generation of gene-edited clone embryos. A. Process of somatic cell nuclear transfer. Scale bar $=100 \mu \mathrm{m}$. B. Embryos at different stages of development. Scale bar $=100 \mu \mathrm{m}$. C. Cleavage rate of donor cells treated with RS-1 and NU7441. D. Ratio of the cloned embryos at different developmental stages from donor cells treated with RS- 1 and NU7441. 


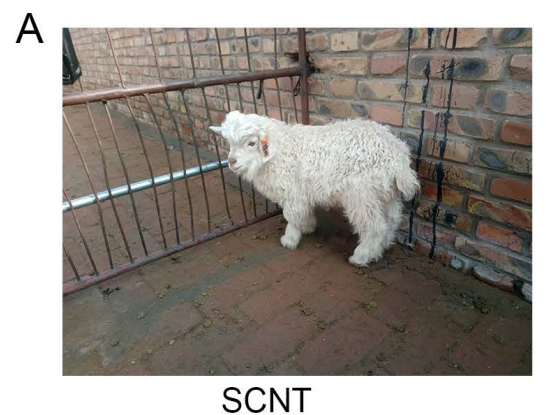

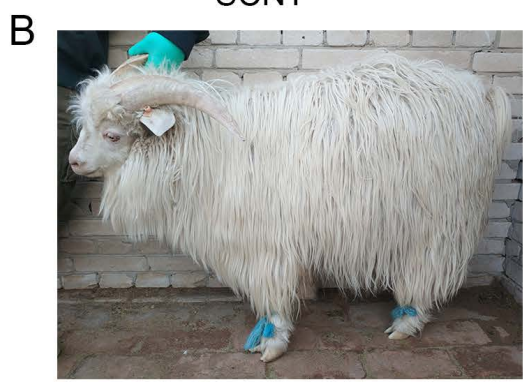

SCNT

C

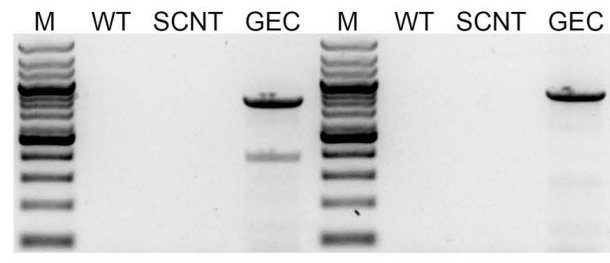

F

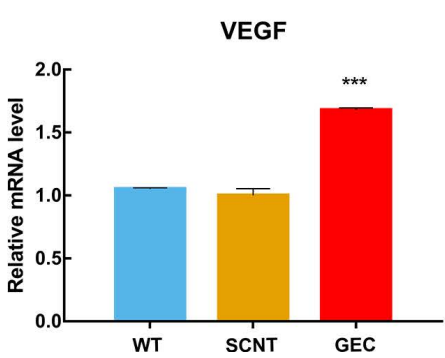

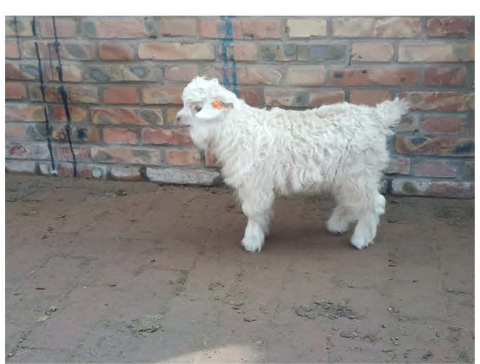

GEC

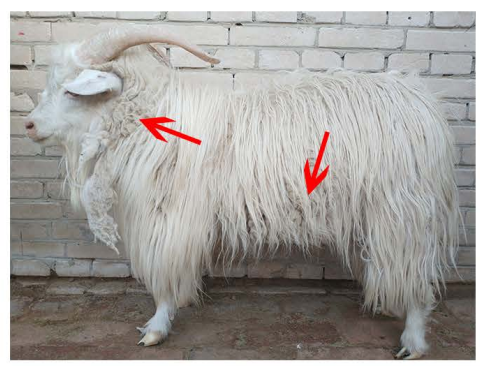

GEC

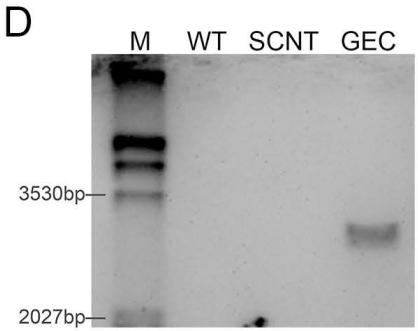

G

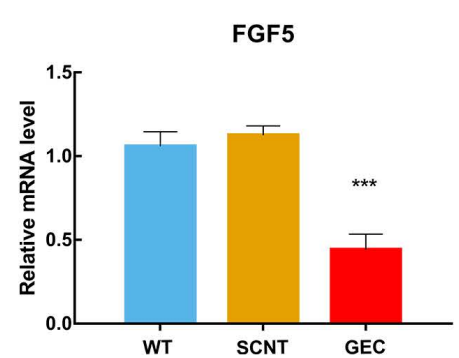

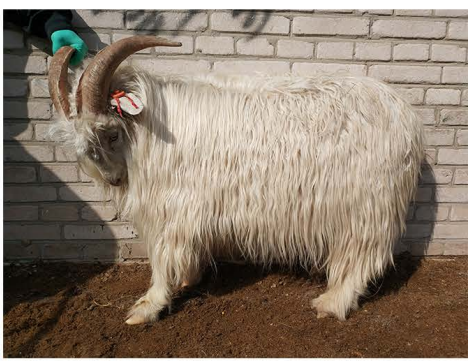

WT

E

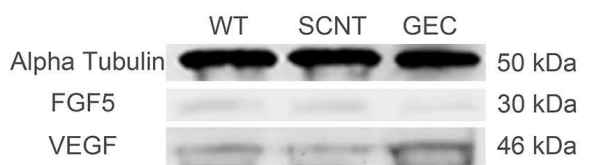

$\mathrm{H}$

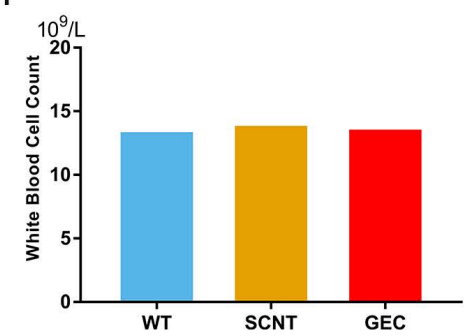

Figure 4. VEGF knock-in Cashmere goats and its identification and detection. A. Images of 1-month-old SCNT and GEC. B. Images of 15-month-old SCNT, GEC, and WT. Red arrows indicate tufts of cashmere. C. Spanning the homologous arms PCR identification results of WT, SCNT and GEC. D. Southern blot identification results of SCNT, GEC, and WT. E. VEGF and FGF5 expression in skin tissues of WT, SCNT and GEC by Western blot. F, G. RT-PCR was used to detect the expression of VEGF and FGF5 in skin tissues of S WT, SCNT and GEC. H. Routine blood tests were performed on WT, SCNT and GEC, and the number of WBC was counted.

\section{Acquisition of cloned cashmere goats}

There were two types of donor cells in the SCNT: mutated cell colonies with VEGF integration in the FGF5 site and GFFs from the same source of embryos without gene editing. A total of 97 2-cell embryos, 73 4-cell embryos, and 25 8-cell embryos were obtained when the gene-edited cells were used as donor cells. They were transplanted into 69 recipient goats, and after approximately 150 days of gestation, a cashmere goat named gene-edited clone (GEC) was born on March 4, 2018 (Figure 4A). A total of 75 2-cell embryos, 70 4-cell embryos, and 21 8-cell embryos of non-gene-edited cells were obtained as donor cells. They were transplanted into 62 recipient goats, and a cashmere goat named SCNT was born on February 23, 2018 (Figure 4A).

\section{Characterisation of VEGF knock-in cashmere goat}

After GEC and SCNT were born, their blood samples were collected for genome extraction, and PCR and electrophoresis were performed. Spanning the upstream and downstream homologous arms PCR results as shown in the Figure $4 \mathrm{C}$, and the Sanger sequencing was correct. To further verify the PCR results, we performed Southern blot analysis. The genomes of GEC, SCNT, and wild type (WT) were treated with the restriction enzymes Xholl and NdelI, and exposed after electrophoresis, membrane transfer, 
and hybridisation. Only GEC showed a specific band of 3,168 bp (Figure 4D). The results of PCR and Southern blot indicated that GEC realised the integration of VEGF at the FGF5 site. We then measured the expression levels of VEGF and FGF5 in skin tissues of cashmere goats. The results showed that the expression level of VEGF was upregulated at both the transcriptional and protein levels, whereas the expression level of FGF5 was downregulated (Figure 4E-G).

No difference from other cashmere goats was observed in GEC at the age of 1 month. At the age of 15 months, after a hair follicle growth cycle, the cashmere synthesis of GEC was more vigorous than those of SCNT or WT, and clusters of cashmere could be clearly seen on the shoulders, neck, and abdomen (Figure 4B). Histological analysis revealed that the secondary hair follicle diameter of GEC seemed to have increased (Figure 5A). We found that it was significantly increased after statistical analysis of different sections (Figure 5B).

In addition, previous work suggested the overexpression of VEGF in the skin caused inflammation [30]. We were also very concerned about the health of gene-edited cashmere goat, so the blood routine test was carried out, and the white blood cell count was shown in Figure $4 \mathrm{H}$. The reference value of white blood cell count in goat is $11.32-15.08 \times 10^{9} / \mathrm{L}$. This result suggested that VEGF does not cause inflammation in the gene-edited goat.

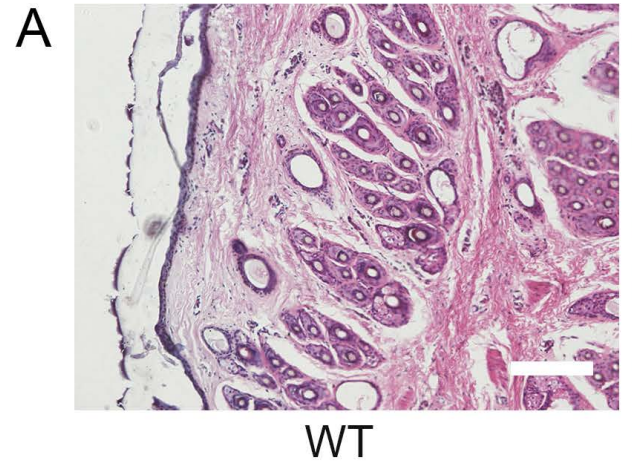

B

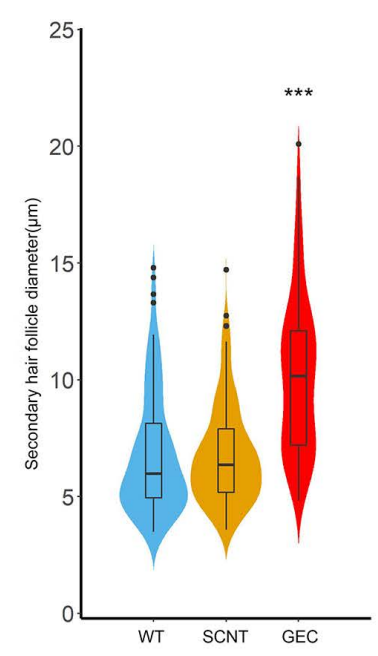

E

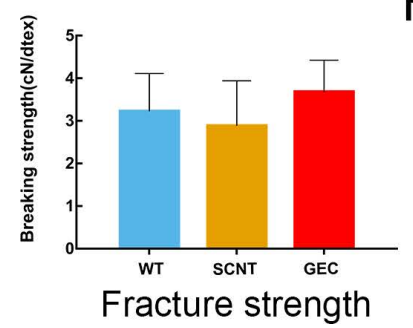

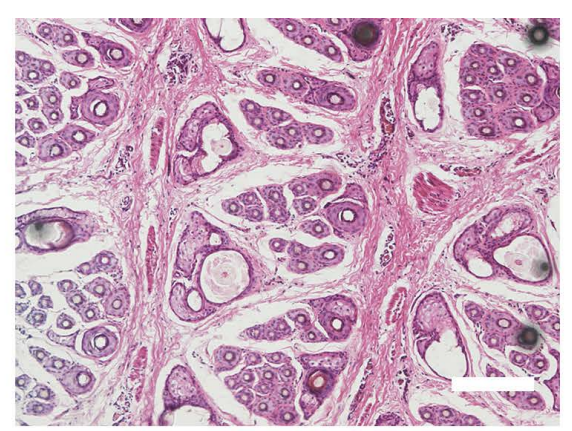

SCNT

C

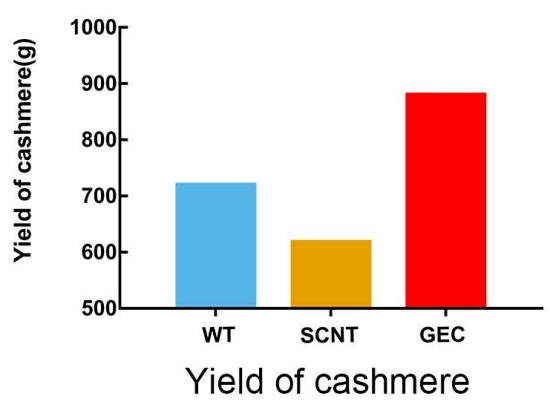

G

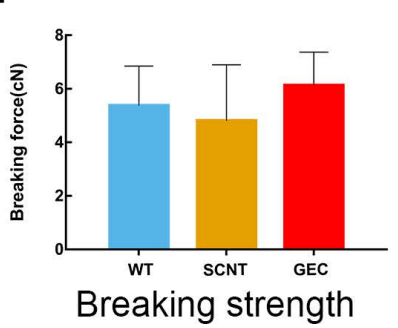

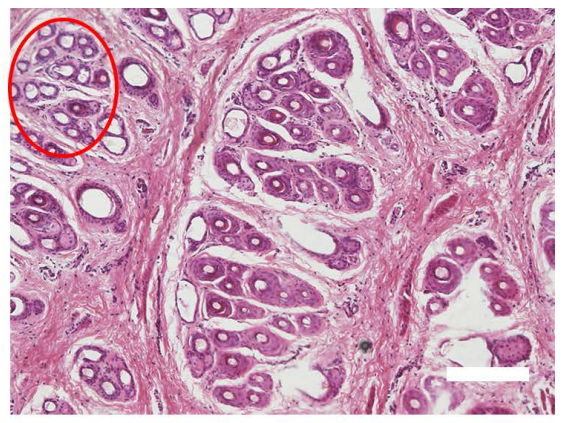

GEC

D

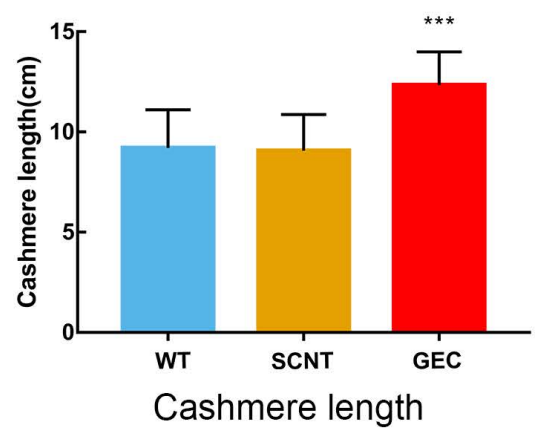

$\mathrm{H}$

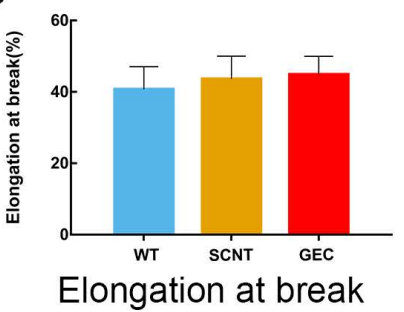

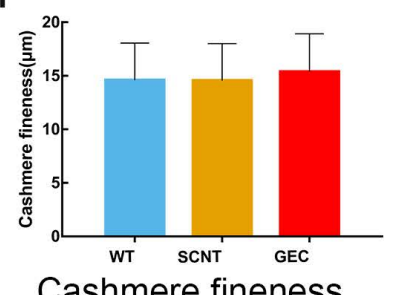

Cashmere fineness

Figure 5. Skin tissue sections and cashmere quality detection. A. Paraffin sections and H\&E staining of WT, SCNT and GEC. Diameter of the secondary follicle in the red circle increased. Scale bar $=100 \mu \mathrm{m}$. B. Secondary hair follicle diameter statistics of WT, SCNT and GEC. C-H. Cashmere yield, cashmere length, fracture strength, breaking strength, elongation at break, and cashmere fineness, respectively, of WT, SCNT and GEC. 
A

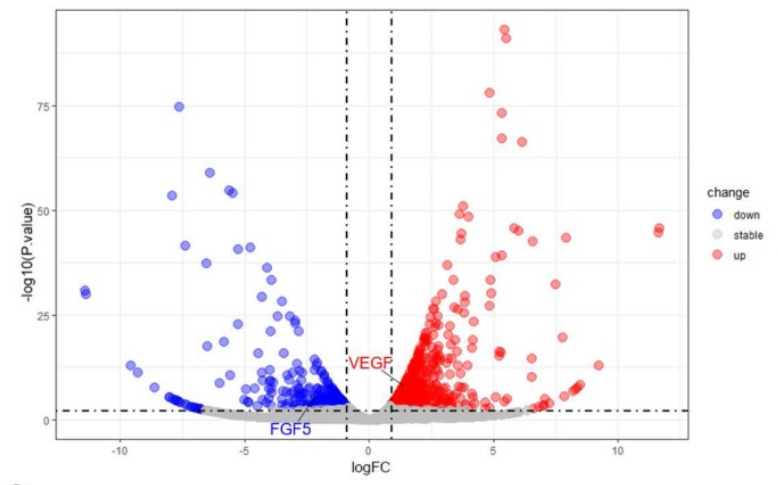

C

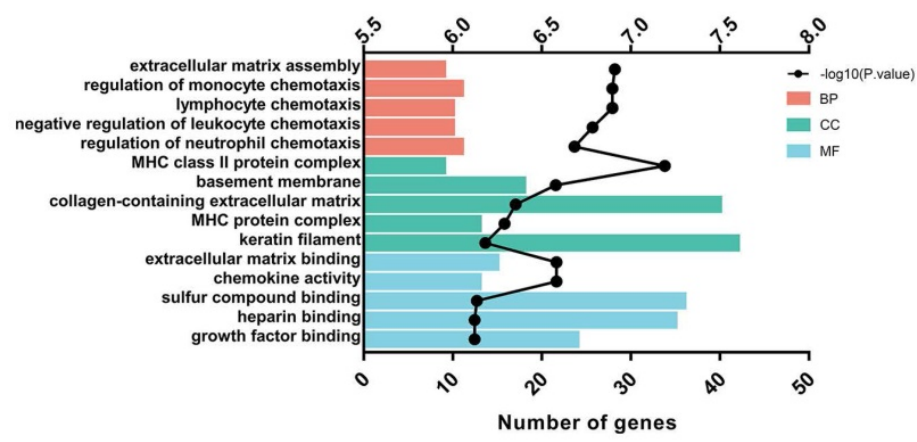

B

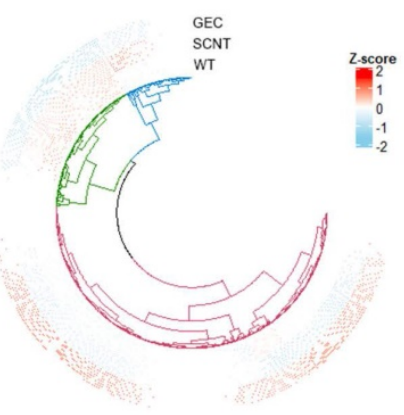

D

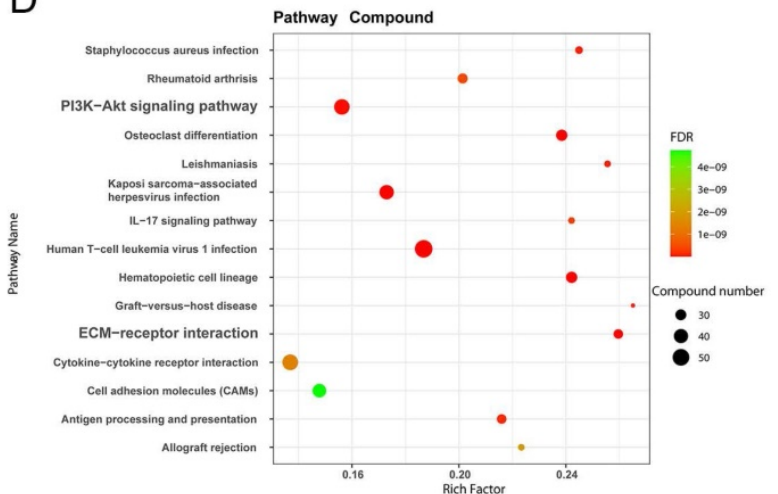

Figure 6. Skin transcriptome sequencing result of cashmere goats. A. Volcano diagram of transcriptome sequencing. The $X$-axis is the log2FC value, and the $Y$-axis is $-\log 10$ (P. value). There were 843 genes upregulated and 403 genes downregulated in GEC. B. Cluster analysis of gene expression patterns was performed for all detected genes. C. GO enrichment analysis was performed for all the differentially expressed genes. The $Y$-axis is the GO term. The top X-axis is -log10 (P. value) and the bottom X-axis is the number of genes in each GO term. D. KEGG enrichment analysis was performed on the differentially expressed genes. We show 15 KEGG pathways with minimum FDR values. The size of the dot represents the number of genes in each pathway.

\section{Determination of yield and quality of cashmere}

The value of cashmere depends greatly on the length and fineness of its fibres. Therefore, we measured the related indexes of the GEC cashmere. After collecting and weighing the cashmere, the GEC cashmere yield seemed to be higher than the SCNT or WT cashmere yields (Figure 5C). However, as only 1 year's data were collected, no one-way ANOVA was conducted. The cashmere fibre length of GEC hairs had increased by approximately $30 \%$ (Figure 5D). The results indicated that the downregulation of FGF5 prolonged the growth period of hair follicles and increased the length of the cashmere. The mechanical properties of cashmere are important to the textile industry, so we examined the fracture strength, breaking strength (Figure 5E-H), elongation at break, and fineness of the GEC cashmere, and found no significant differences from those of the SCNT and WT cashmere. The above results showed that the increase in yield and fibre length of the cashmere produced by GEC did not affect its quality.

\section{Overview of transcriptome data}

To reveal the mechanism by which VEGF and
FGF5 promote hair growth, transcriptome, proteome, and metabolome studies were conducted on goat skin tissues. Transcriptomic analysis of the three samples was completed, and a total of $22.33 \mathrm{~Gb}$ of Clean Data was obtained. The Clean Data of each sample reached more than $6.59 \mathrm{~Gb}$, and the percentage of the Q30 base was over $94.01 \%$. The sequence alignment rate ranged from $95.02-96.1 \%$. In this analysis, a total of 22,167 expressed genes were detected, including 19,266 known genes and 2,901 new genes. Finally, 843 genes were upregulated and 403 genes were downregulated. Volcano mapping was performed on all the detected genes, and VEGF and FGF5 were both DEGs (Figure 6A). A cluster analysis of the 1,246 DEGs showed that the clusters of SCNT and WT were intact (Figure 6B). Gene ontology enrichment analysis was performed on all the DEGs, and 24 genes were enriched in growth factor binding and extracellular matrix (ECM) assembly functions (Figure 6C). Consistent with this, KEGG revealed that the DEGs were significantly enriched in the PI3K-AKT signalling pathway and ECM-receptor interactions (Figure 6D). These results suggested that VEGF and FGF5 altered cell growth status by affecting ECMreceptor responses and cell growth factor binding. 

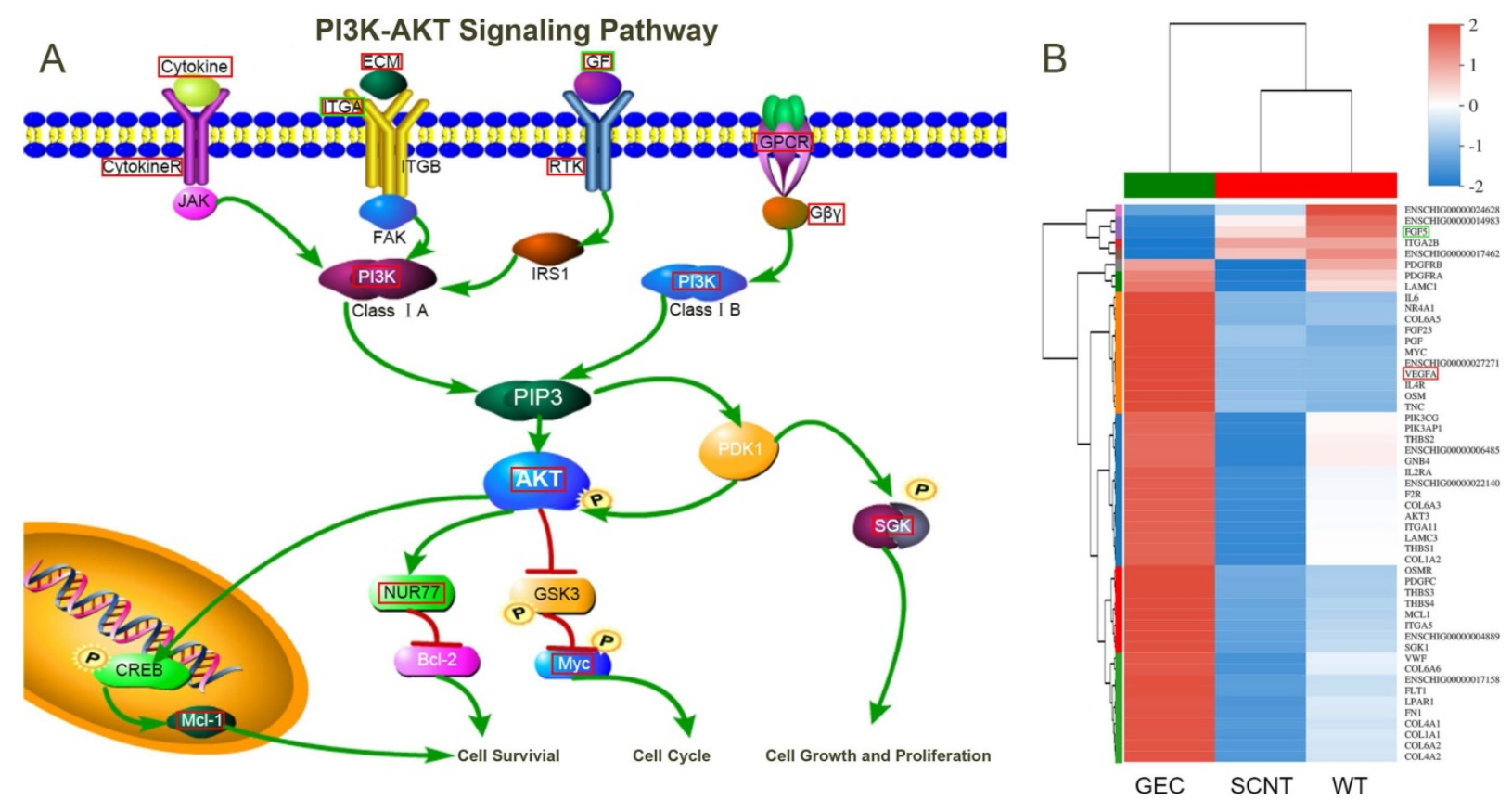

Figure 7. PI3K-AKT Signaling Pathway. A. Schematic diagram of key factors in the PI3K-AKT signalling pathway. B. Cluster analysis was performed on the genes enriched in the PI3K-AKT signalling pathway. The red box indicates upregulated, and the green box indicates downregulated genes.

\section{DEGs enriched in the PI3K-Akt signaling pathway and ECM-receptor interaction}

A total of 51 DEGs were enriched in the PI3K-AKT signalling pathway. A clustering heat map was drawn for these 51 DEGs (Figure 7B) and the pathway was visualised (Figure 7A). In the figure, the red boxes represent upregulated gene expression, and the green boxes represent downregulated gene expression. Changes in the expression levels of cell membrane receptors, such as CytokineR, ITGA, RTK, and GPCR, and their ligands caused a cascade of intracellular amplification of signals acting on the key molecule, AKT. Finally, cell growth, proliferation, cycle, and survival were regulated by downstream McL-1, Bcl-2, Myc, and SGK.

In addition, DEGs were also enriched in ECM-receptor interactions. These were mainly ligands located in the ECM (Figure 8A). We visualised the protein-protein interaction network for the DEG-translated proteins enriched in the PI3K-AKT signalling pathway and ECM-receptor interactions, and the core proteins were fibronectin-1 (FN1), VEGFA, collagen type I alpha 1 (COL1A1), interleukin-6 (IL6), cluster of differentiation-4 (CD4), and platelet-derived growth factor receptor beta (PDGFRB) (Figure 8B).

\section{Correlation analysis of proteome and metabolome}

A total of 178 differentially expressed proteins was detected by proteomic sequencing, and KEGG functional annotation was performed (Figure 9A). A total of 1,706 differential metabolites was detected in the metabolome (122 of which were named), and KEGG functional annotation was performed (Figure 9B). A Venn diagram was drawn for the pathways annotated to the differential proteins and metabolites (Figure 9C), and 12 common annotated pathways were obtained (Figure 9D). Among them, cell growth and death, signalling molecules and interactions, and the signal transaction profile verified our transcriptome sequencing results.

\section{Discussion}

DSB causes endogenous repair mechanisms in cells, such as non-homologous end joining (NHEJ), and HDR [31]. NHEJ is the forced joining of two fractured DNA strands, and the repair process results in the insertion or deletion of nucleotide fragments, which destroys the open reading frame of the gene at the junction and produces mutations. Destroying the functional components of a gene or genome can help us to uncover its functions and mechanisms [32]. The precise insertion or deletion of exogenous genes or regulatory sequences can be realised using HDR, which is the most dependable repair mechanism for genome site-specific integration [33].

The probability of cells using HDR for DNA repair is low (approximately $0.5 \%$ to $20 \%$, depending on a variety of factors including cell type and cell state), whereas the probability of antagonistic NHEJ can be as high as $80 \%$ [34, 35]. Therefore, we used small molecules to improve the efficiency of gene knock-in by promoting HDR and inhibiting NHEJ. 
A

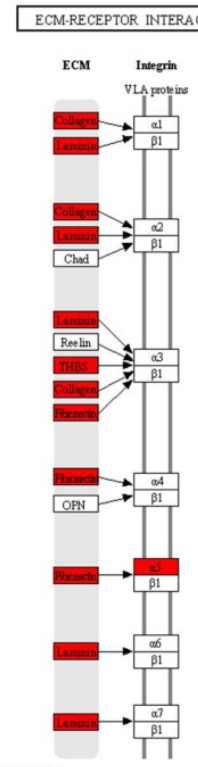

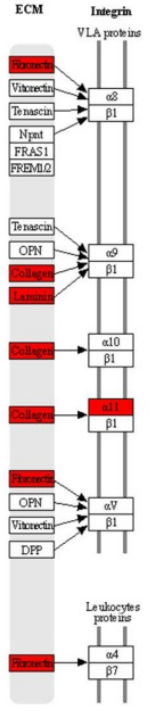
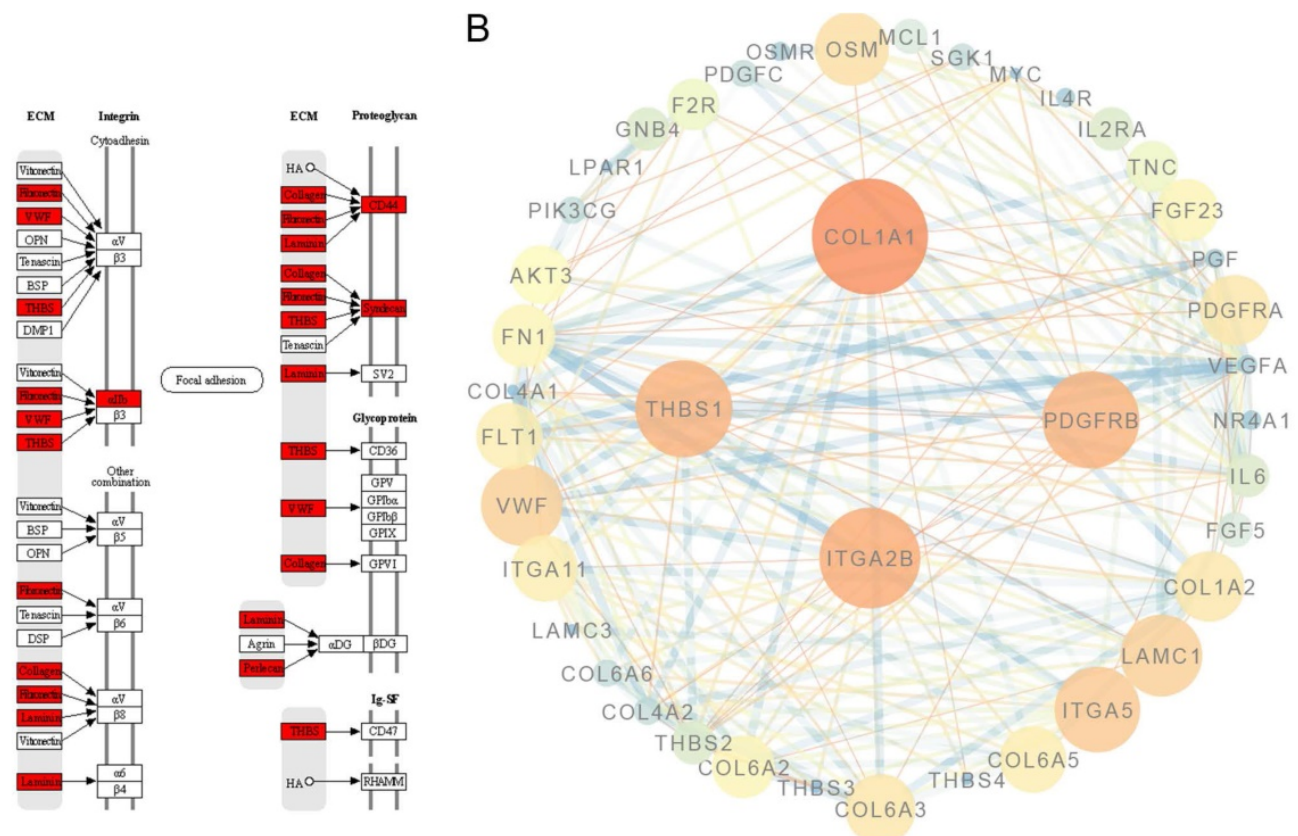

Figure 8. Protein-protein interaction in the PI3K-AKT signalling pathway and ECM-receptor interactions. A. ECM-receptor interaction pathway was adapted from KEGG; the red box indicates the upregulated genes. B. Protein-protein interaction network of genes enriched in the PI3K-AKT signalling pathway and ECM-receptor interactions. The core proteins were FNI, VEGFA, COL1A1, IL6, CD4, and PDGFRB.

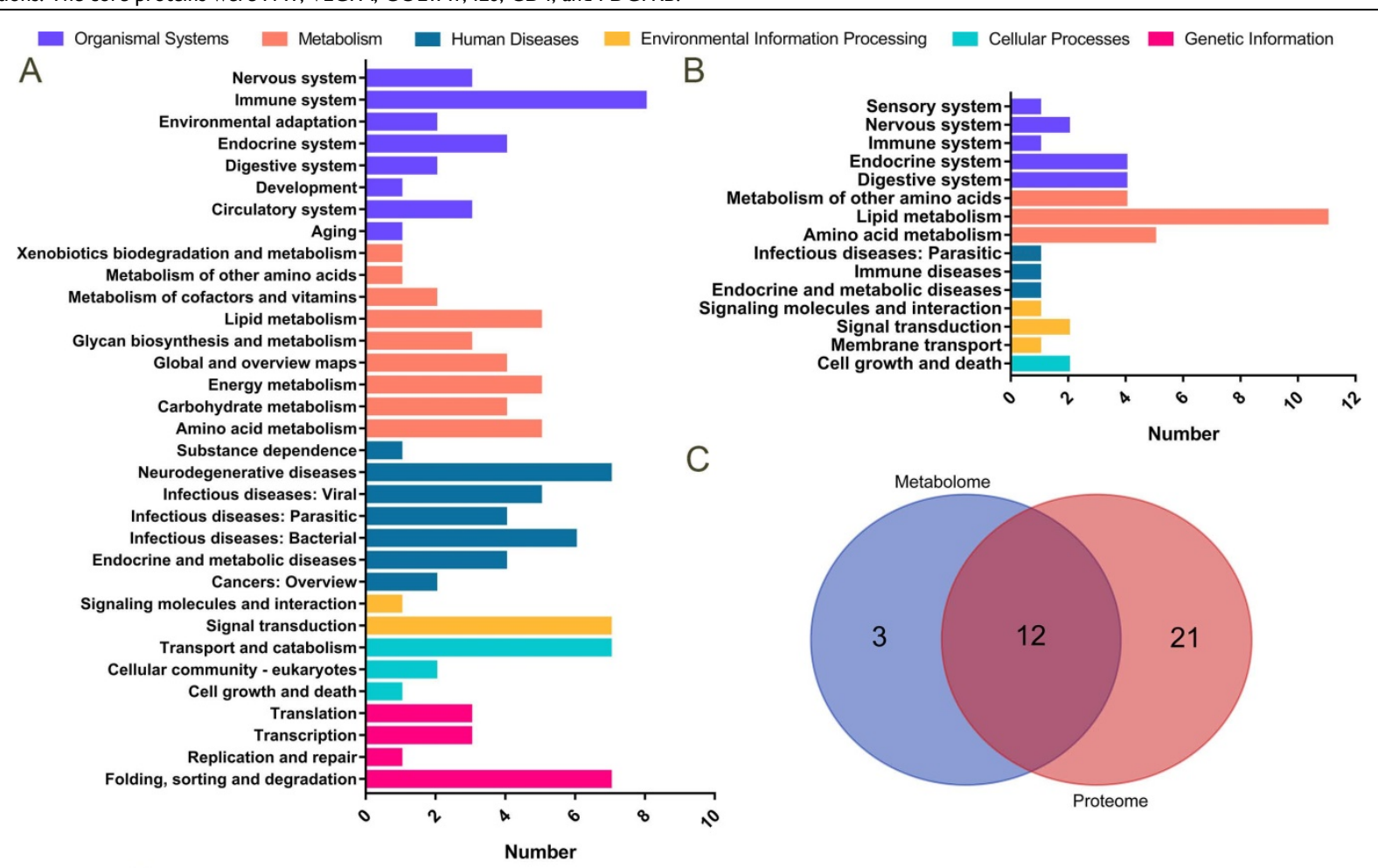

$\mathrm{D}$

\begin{tabular}{|c|c|}
\hline \multicolumn{2}{|c|}{ KEGG annotation of metabolome \& proteome in common } \\
\hline Endocrine and metabolic diseases & Lipid metabolism \\
\hline Nervous system & Endocrine system \\
\hline Cell growth and death & Amino acid metabolism \\
\hline $\begin{array}{c}\text { Signaling molecules and interaction } \\
\text { Infectious diseases: Parasitic }\end{array}$ & Digestive system \\
\hline Immune system & Signal transduction \\
\hline
\end{tabular}

Figure 9. Proteomics and metabolomics analysis. A. KEGG functional annotation was performed on 178 differentially expressed proteins in proteomics sequencing. The $\mathrm{Y}$-axis is the name of the KEGG metabolic pathway, and the X-axis is the number of proteins annotated to this pathway. B. KEGG functional annotation was performed on 122 differentially expressed metabolites in metabolome sequencing. C. Venn diagrams for the annotated pathways in metabolome and proteome sequencing. D. Table of 12 KEGG pathways annotated both in proteome and metabolome sequencing. 
There have been many studies on small molecules promoting HDR efficiency, but the performance of the same inhibitor differs among different species and cell types [36]. Here, the HDR activator RS-1, and NHEJ inhibitor NU7441 were selected for the experiment. We found that the efficiency of HDR was significantly improved when RS-1 and NU7441 were used together.

Although SCNT is the only known method of obtaining the totipotency for somatic cells so far, owing to various epigenetic disorders in reprogramming, the reprogramming of somatic cells is not complete, resulting in extremely low developmental potential for nuclear transplantation embryos [37]. We found that RS-1 and NU7441 treatment of GFFs did not affect the embryo cleavage rate or ratio of cloned embryos at different developmental stages when the treated cells were used as donor cells. Therefore, RS-1 and NU7441 can be used in the generation of genetically edited goats.

However, only two goats were born from 131 recipients, and the live birth rate was only $1.5 \%$. We speculate that this might be caused by the incomplete reprogramming of donor cells, resulting in the retardation or failure of embryo development in the early stage of transplantation to recipients. During preparation of the world's first somatically cloned monkey, the mRNA36 of histone lysine demethylase $4 \mathrm{D}$ was injected into the fused, activated, and reconstructed embryo to remove some of the key epigenetic modifications in the donor nuclear genes, significantly improving reprogramming efficiency [38]. In subsequent experiments, we can also improve the efficiency of reprogramming and remove epigenetic modifications to improve the birth rate of individuals.

The safety of gene-edited organisms is controversial. While pursuing efficiency and practicality, researchers are increasingly concerned with the possibility of causing harm to human beings and the ecological environment. From the perspective of biological safety, we did not introduce resistance genes or fluorescent proteins in our preparations. In addition, we linked the KAP6.1 promoter upstream of the VEGF gene. KAPs are a class of hair folliclespecific proteins [39], and their promoters lead to the follicle-specific expression of downstream genes [40]. In this study, the KAP6.1 promoter was used to initiate the transcription of VEGF in hair follicles, which not only promoted the development of cashmere follicles but also avoided the expression of VEGF in other tissues, which might have affected other physiological activities.

We found that VEGF expression was upregulated and FGF5 was downregulated in the skins of cashmere goats. This means that we achieved the goal of VEGF overexpression and FGF5 knockout. FGF5 downregulation prolonged the anagen phase of hair and increased the length of cashmere fibres produced by GEC. Meanwhile, upregulated VEGF expression promoted the proliferation of cells that formed secondary hair follicles and increased their diameter. Furthermore, vigorous cashmere synthesis was promoted and clustering characteristics improved. Ultimately, cashmere yield was greatly improved without affecting its quality.

Previous studies have shown that VEGF can induce the differentiation of hair follicle stem cells into endothelial cells, thus promoting angiogenesis [13]. It has been reported that after FGF5 knockout, the ratio of secondary hair follicles (SHF) to primary hair follicles (PHF) and the length of cashmere fibres increased [24]. In our gene-edited goat, we did not find an increase in the SHF/ PHF ratio, despite the fact that we statistically analysed a large number of histological sections. Since the specific molecular mechanism remained unclear, we used highthroughput sequencing technology to study it.

We found that FN1, VEGFA, COL1A1, IL6, CD4, and PDGFRB were the key components in the protein-protein interaction network. FN1 is involved in cell adhesion and migration processes, including embryogenesis, wound healing, blood coagulation, host defence, and metastasis [41]. COL1A1 is a fibril-forming collagen found in most connective tissues and is abundant in bone, cornea, dermis, and tendon tissues. COL1A1 is associated with a particular type of skin tumour (dermatofibrosarcoma protuberans) resulting from unregulated expression of growth factors [42]. IL6 is primarily produced at sites of acute and chronic inflammation, where it is secreted into the serum and induces a transcriptional inflammatory response through IL6 receptor alpha [43]. CD4 is a cell-surface glycoprotein involved in cell-cell interactions and cell adhesion and migration [44]. These key molecules are involved in cell interactions, adhesions, and migrations and promote the synthesis of cashmere.

In conclusion, we found that a combination of RS-1 and NU7441 could improve the efficiency of CRISPR/Cas9-mediated HDR, without affecting the embryo status. Based on these findings, we obtained a cashmere goat that integrated the VEGF gene at the FGF5 site. The diameter of the secondary hair follicles and the length of the cashmere fibres of this gene-edited cashmere goat were improved. The upregulation of $V E G F$ and the downregulation of FGF5 affected cell cycle, proliferation, and vascular tone through the PI3K-AKT signalling pathway, ECM-receptor interactions resulted in the gene-edited 
cashmere goat showing impressive cashmere performance. The results of this study provide a basis for the efficient generation of gene knock-in animals. It also provides an animal model for the study of the hair growth-promoting mechanisms in cashmere goats.

\section{Abbreviations}

VEGF: vascular endothelial growth factor; FGF5: fibroblast growth factor 5; DSB: double-strand break; CRISPR: clustered regulatory interspaced short palindromic repeat; SCNT: somatic cell nuclear transfer; GFFs: goat foetal fibroblast cells; DMEM: dulbecco's modified eagle's medium; HDR: homologous-directed repair; HA: homology arm; FACS: fluorescence activated cell sorting; Kap: keratin-associated protein; PCR: polymerase chain reactions; DEGs: differentially expressed genes; GO: Gene Ontology; KEGG: Kyoto encyclopedia of genes and genomes; SD: standard deviation; GEC: gene-edited clone; ECM: extracellular matrix; FN1: fibronectin-1; COL1A1: collagen type I alpha 1; IL6: interleukin-6; CD4: cluster of differentiation-4; PDGFRB: platelet-derived growth factor receptor beta; NHEJ: non-homologous end joining.

\section{Supplementary Material}

Supplementary tables.

http://www.ijbs.com/v17p1026s1.pdf

\section{Acknowledgements}

This work was supported by the Science and Technology Innovation Guided Project in Inner Mongolia Autonomous Region (KCBJ2018003 to D.J.L.); Science and Technology Major Project of Inner Mongolia Autonomous Region of China to the State Key Laboratory of Reproductive Regulation and Breeding of Grassland Livestock (30500-5203901 to D.J.L.; 30500-518390215 to F.H.); Natural Science Foundation of Inner Mongolia Autonomous Region (2019BS03026 to F.H.); The Funding of Top Disciplines Development in Inner Mongolia University, Research Foundation for Advanced Talents of Inner Mongolia University (30500-5185141 to F.H.). Thanks to the Inner Mongolia YiWei White Cashmere Goat limited liability company for providing the recipient goats. We would like to thank Editage for English language editing.

\section{Author contributions}

Xiao $\mathrm{Hu}$ and Fei Hao performed the experiments. Xiaocong Li and Zhiyuan Xun conceived the idea. Yuan Gao and Bingxu Ren wrote the manuscript. Hao Liang and Ming Cang revised the manuscript. Fei Hao and Dongjun Liu provided funding support. All authors reviewed the manuscript.

\section{Competing Interests}

The authors have declared that no competing interest exists.

\section{References}

1. Dai $Y$, Vaught TD, Boone J, et al. Targeted disruption of the a1,3-galactosyltransferase gene in cloned pigs. Nature biotechnology. 2002; 20: 251-5.

2. Lai L, Kolber-Simonds D, Park KW, et al. Production of alpha -1,3-Galactosyltransferase Knockout Pigs by Nuclear Transfer Cloning. Science. 2002; 295 : 1089-92.

3. Capecchi MR. The new mouse genetics: Altering the genome by gene targeting. Trends in Genetics. 1989; 5: 70-6.

4. McCreath KJ, Howcroft J, Campbell KH, et al. Production of gene-targeted sheep by nuclear transfer from cultured somatic cells. Nature. 2000; 405:1066-9.

5. Barrangou R, Fremaux C, Deveau H, et al. CRISPR provides acquired resistance against viruses in prokaryotes. Science. 2007; 315: 1709.

6. Shulman MJ. Model for wandering restriction enzymes. Nature. 1974; 252: 76-8.

7. Fu Y, Foden JA, Khayter C, et al. High-frequency off-target mutagenesis induced by CRISPR-Cas nucleases in human cells. Nature biotechnology. 2013; 31: 822-6

8. Ma Y, Zhang L, Huang X. Genome modification by CRISPR/Cas9. FEBS J. 2014; 281: 5186-93.

9. Makarova KS, Wolf YI, Alkhnbashi OS, et al. An updated evolutionary classification of CRISPR-Cas systems. Nature reviews Microbiology. 2015; 13: 722-36.

10. Gaj T, Gersbach CA, Barbas CF 3rd. ZFN, TALEN, and CRISPR/Cas-based methods for genome engineering. Trends in biotechnology. 2013; 31: 397-405.

11. Nam JO, Son HN, Jun E, et al. FAS1 Domain Protein Inhibits VEGF(165)-Induced Angiogenesis by Targeting the Interaction between VEGFR-2 and alpha v beta 3 Integrin. Molecular Cancer Research. 2012; 10: 1010-20

12. Makarevich PI, Boldyreva MA, Gluhanyuk EV, et al. Enhanced angiogenesis in ischemic skeletal muscle after transplantation of cell sheets from baculovirus-transduced adipose-derived stromal cells expressing VEGF165. Stem Cell Research \& Therapy. 2015; 6: 204.

13. Quan R, Du W, Zheng X, et al. VEGF165 induces differentiation of hair follicle stem cells into endothelial cells and plays a role in in vivo angiogenesis. Journal of Cellular and Molecular Medicine. 2017; 21: 1593-604.

14. Li W, Lu ZF, Man XY, et al. VEGF upregulates VEGF receptor-2 on human outer root sheath cells and stimulates proliferation through ERK pathway. Molecular biology reports. 2012; 39: 8687-97.

15. Li W, Man XY, Li CM, et al. VEGF induces proliferation of human hair follicle dermal papilla cells through VEGFR-2-mediated activation of ERK. Experimental cell research. 2012; 318: 1633-40.

16. Stenn KS, Paus R. Controls of Hair Follicle Cycling. Physiological Reviews. 2001; 81: 449-494.

17. Lihua $\mathrm{Su}, \mathrm{Hua} \mathrm{Li}$, Rigan $\mathrm{Xu}$, et al. Molecular regulation of hair follicles. Chemistry of life. 2011; 31: 455-60

18. Li CX, Jiang MS, Chen SY, et al. Correlation analysis between single nucleotide polymorphism of FGF5 gene and wool yield in rabbits. Hereditas. 2008; 30: 893-9.

19. Kehler JS, David VA, Schäffer AA, et al. Four Independent Mutations in the Feline Fibroblast Growth Factor 5 Gene Determine the Long-Haired Phenotype in Domestic Cats. Journal of Heredity. 2007; 98: 555-66.

20. Cadieu E, Neff MW, Quignon P, et al. Coat variation in the domestic dog is governed by variants in three genes. Science. 2009; 326: 150-3.

21. Dierks C, Momke S, Philipp U, et al. Allelic heterogeneity of FGF5 mutations causes the long-hair phenotype in dogs. Animal genetics. 2013; 44: 425-31.

22. Legrand R, Tiret L, Abitbol M. Two recessive mutations inFGF5are associated with the long-hair phenotype in donkeys. Genetics Selection Evolution. 2014; 46: 65 .

23. Chen Z, Wang Z, Xu S, et al. Characterization of hairless (Hr) and FGF5 genes provides insights into the molecular basis of hair loss in cetaceans. Bmc Evolutionary Biology. 2013; 13:34

24. Wang X, Cai B, Zhou J, et al. Disruption of FGF5 in Cashmere Goats Using CRISPR/Cas9 Results in More Secondary Hair Follicles and Longer Fibers. PloS one. 2016; 11: e0164640.

25. Song J, Yang D, Xu J, et al. RS-1 enhances CRISPR/Cas9- and TALENmediated knock-in efficiency. Nat Commun. 2016; 7: 10548.

26. Robert F, Barbeau M, Éthier S, et al. Pharmacological inhibition of DNA-PK stimulates Cas9-mediated genome editing. Genome Medicine. 2015; 7: 93.

27. Diermeier-Daucher S, Clarke ST, Hill D, et al. Cell type specific applicability of 5-ethynyl-2'-deoxyuridine (EdU) for dynamic proliferation assessment in flow cytometry. Cytometry A. 2010; 75a: 535-46. 
28. Smith, Paul, J. EdU and BrdU incorporation resolve their differences. Cell Cycle. 2016; 15:1527-8.

29. Maurice L, Adams FF, Michelle N, et al. Refined sgRNA efficacy prediction improves large- and small-scale CRISPR-Cas9 applications. Nucleic Acids Res. 2018; 46:1375-85.

30. Xia YP, Li B, Hylton D, et al. Transgenic delivery of VEGF to mouse skin leads to an inflammatory condition resembling human psoriasis. Blood. 2003; 102: 161-8.

31. Featherstone C, Jackson SP. DNA Double-strand Break Repair. Current Biology. 1999; 9: 759-61.

32. Shalem O, Sanjana NE, Hartenian E, et al. Genome-scale CRISPR-Cas9 knockout screening in human cells. Science. 2014; 343: 84-7.

33. Chatterjee N, Walker GC. Mechanisms of DNA damage, repair, and mutagenesis. Environmental and molecular mutagenesis. 2017; 58: 235-63.

34. Doudna JA, Charpentier E. The new frontier of genome engineering with CRISPR-Cas9. Science. 2014; 346: 1258096.

35. Doetschman T, Georgieva T. Gene Editing With CRISPR/Cas9 RNA-Directed Nuclease. Circulation Research. 2017; 120: 876-94.

36. Zhang JP, Li XL, Li GH, et al. Efficient precise knockin with a double cut HDR donor after CRISPR/Cas9-mediated double-stranded DNA cleavage. Genome biology. 2017; 18: 35

37. Niemann H. Epigenetic reprogramming in mammalian species after SCNT-based cloning. Theriogenology. 2016; 86: 80-90.

38. Liu Z, Cai Y, Wang Y, et al. Cloning of Macaque Monkeys by Somatic Cell Nuclear Transfer. Cell. 2018; 174:245.

39. Yang Z, Cui K, Zhang Y, et al. Transcriptional regulation analysis and the potential transcription regulator site in the extended KAP6.1 promoter in sheep. Molecular biology reports. 2014; 41: 6089-96.

40. McLaren RJ, Rogers GR, Davies KP, et al. Linkage mapping of wool keratin and keratin-associated protein genes in sheep. Mammalian Genome. 1997; 8: 938-40.

41. Kuczyk, M. Fibronectin 1 protein expression in clear cell renal cell carcinoma. Oncology Letters. 2012; 3: 787-90.

42. Steffens S, Schrader AJ, Vetter G, et al. Fibronectin 1 protein expression in clear cell renal cell carcinoma. Oncology Letters. 2012; 3: 787-90.

43. Babon JJ, Stockwell D, Dirago L, et al. Membrane-Associated RING-CH (MARCH) proteins down-regulate cell surface expression of the interleukin-6 receptor alpha chain (IL6Ra). Biochemical Journal. 2019; 476:2869-2882.

44. König R, Ashwell G, Hanover JA. Glycosylation of CD4. Tunicamycin inhibits surface expression. J Biol Chem. 1988; 263: 9502-7. 